\title{
Forme della realtà, forme della conoscenza. Verso un'estetica della formazione dei ricercatori
}

\section{Antonia Chiara Scardicchio}

Università degli Studi di Foggia, Dipartimento di Studi Umanistici

doi: 10.7358/ecps-2013-008-scar

ac.scardicchio@unifg.it

\section{FORMS OF REALITY, FORMS OF KNOWLEDGE. TOWARDS AESTHETICS OF RESEARCHER TRAINING}

\begin{abstract}
The paper starts from a reflection on the two forms of human knowledge - analytic and symbolic - and then probes their joint use in the training of researchers of any discipline or academic field. Scientific knowledge is thus seen as irreducibly based on discipline crossing, towards the proposal of an aesthetics of researcher training, where "aesthetics" corresponds to «systemic sensitivity", indicated by Bateson as that «ecological» knowledge which can grasp conjunctions-crossings-links between knowledge, languages and methods from different domains, towards the construction of an «interdisciplinary science that deals with analogies». The goal is that «New Science» - which Morin takes from Vico - characterized by abduction, serendipity, the valorisation of elements historically considered as lateral in research, where "the ways of post-Darwin thinking» translate into aesthetic educational designs: binocular, complex and systemic in nature. In this sense, the capability of «double description" - the zig zag epistemology indicated by Bateson - outlines the isomorphism between the skills of the researcher-of any discipline-and aesthetic posture.
\end{abstract}

Keywords: Aesthetic, Discipline crossing, Scientific knowledge, Systemic vision, Training for research. 


\section{LA Mitologia È UMANA}

La mitologia è umana. La computazione animale ignora il mito e può con ciò sembrare più razionale della nostra cogitazione. A lungo si è creduto che il mito fosse un'illusione primitiva, nata da un uso ingenuo del linguaggio. E invece occorre capire che il mito appartiene non tanto a un pensiero arcaico superato quanto a un Archi-Pensiero sempre vivo. Esso deriva da quella che potremmo definire un Archi-Mente, che non vuol dire una mente arretrata bensì una Retro-Mente la quale, conformemente al senso forte del termine Archè, corrisponde alle forze e alle forme originali, iniziali e fondamentali dell'attività cerebro-spirituale, là dove i due pensieri non sono ancora separati [...]. Così la Retro-Mente produce spontaneamente ciò che il mito conferma sistematicamente, cioè la credenza nei segni [...] e più generalmente la credenza che tutto sia segno. (Morin, 1983, pp. 189-190)

La riflessione di Morin catapulta con forza nel bel mezzo di un incrocio: tra scienza ed arte, tra parola ed immagine e, anche, tra chiaro ed opaco. Un crocevia al quale la progettazione pedagogica si ferma e si interroga a proposito dei linguaggi della umana conoscenza e dunque dei linguaggi della formazione.

$\mathrm{E}$ a proposito di poliglossie (Scardicchio, 2012a), insieme alle meditazioni di Feyerabend (1984) e Gadamer (1983), giunge una pagina singolare e significativa del Talmud. È quella in cui si riconosce l'esistenza di due linguaggi per comprendere e abitare il mondo: la halakhà, costituita dalle interpretazioni normative e la haggadà, costituita da storie, parabole, leggende e aneddoti:

L'halakhà ha volto severo, l'haggadà ha volto ridente.

L'una è meticolosa, rigida, esigente, più dura del ferro, è la voce del diritto; l'altra è liberale, indulgente, condiscendente, più molle dell'olio; è la voce della bontà.

L'una è la scorza, il corpo, l'azione; l'altra è l'interno, l'anima, l'intenzione devota.

L'una è l'impero della ragione; l'altra è la dolcezza della poesia, l'impero del sentimento. (Salvarani, 1997, p. 16)

Così le "due anime» sono presentate dal poeta ebraico Chajjim Nachman Bialik e così i rabbini solevano un tempo rispondere a chi domandava loro come conoscere Dio: «Volete fare la conoscenza di Colui che parlò e il mondo fu? Studiate la haggadà!» (ibidem).

Come dire che non già nel linguaggio analitico - che meticolosamente separa, ordina, subordina concetti isolati, spogliati di ambiguità e ridotti a scheletro - si manifesta la "verità», non in quella forma - ordinata, chiara, immobile - che noi vorremmo per essa ma quella - disordinata, sfumata, imprevedibile - che le è propria. E, dunque, non già nell'astrazione risie- 
de la conoscenza ma, anzi, nella ibridazione. Ibridazione intesa non come mostruosità, deviazione, meticciamento contrario alla conoscenza ma, anzi, sua stessa forma, perché forma stessa del mondo (Morelli, 2010). E che è la forma del linguaggio simbolico, isomorfico alla realtà, così come alla mente umana (Lucignani \& Pinotti, 2007).

"Simbolo»: dal greco symbállo, "tenere insieme», significa coniugare, congiungere, legare. La conoscenza simbolica è, difatti, forma di rappresentazione e conoscenza che si sostanzia non nella «separazione da" l'oggetto che si vuole conoscere, o nella scissione tra parti, concetti, campi, ma proprio nell'opposto della conoscenza analitica: ibridare, piuttosto che purificare.

L'interrogazione del simbolo non ci spinge ad annullare il lato oscuro, così da poter dire tutte le parole o illuminare la tenebra, piuttosto è un invito a imparare a dimorare nel fra. [...] Il simbolo è mediatore e Caronte da un lato all'altro della soglia: e solo un pensiero immaginale è capace di entrambi i lati, senza piegarsi allo scandalo della contraddizione; come fin da Goethe è stato compreso, esso realizza l'unità dei contrari, conduce alla conoscenza attraverso la dinamica propria del rinvio tra ciò che è mostrato e ciò a cui il mostrato allude. (Mottana, 2002, pp. 29, 35)

Superare «lo scandalo della contraddizione», rinvenire i nessi tra colui che conosce e il conosciuto, in una relazione ove colui-che-conosce è anche conosciuto, in una danza senza egemonia né presunzione del conoscente: pensare di esistere in-sé, prima e a prescindere dal tessuto nel quale si è irriducibilmente immersi, in quanto corporeità, è la forma illuministica e cartesiana della conoscenza che, piuttosto che alla realtà, è isomorfica alla umana insicurezza.

Nel linguaggio simbolico, altresì, abbandonata l'aristotelica pretesa del ricondurre tutto all'identico - certamente meno pericoloso perché controllabile, meno spaventoso perché prevedibile -, il desiderio di arginare l'inatteso lascia il posto alla capacità di sorprendersi, il che implica per la ratio cartesiana imparare a concedere la parola ad altre ragioni affinché la parola analitica riconosca legittimità ad altre parole: a quelle dell'emisfero destro, che, diversamente dal linguaggio dell'emisfero sinistro, anziché de-finire la realtà, la ricrea mediante le vie della rappresentazione simbolica (De Mendoza, 1996).

\section{IMMAGINO, DUNQUE PENSO}

Tale riconoscimento della crucialità della rappresentazione simbolica e dell'immagine/analogia/metafora nei processi di comprensione dell'umano oggi condiviso in molteplici campi del sapere scientifico - ha una paternità 
più antica in Jung (1965), per il quale l'immaginazione non è fantasticheria o illusione, ma, anzi, parte integrante dei processi di conoscenza e di esperienza:

[...] la «funzione simbolica dell'inconscio» [...] non riveste affatto un carattere meramente "poetico» o astratto, cioè importante ma tutto sommato marginale per l'economia globale dell'individuo. Al contrario, la «funzione simbolica», in quanto principale espressione dell'attività "creativa» rappresenta una funzione fondamentale per il soggetto proprio nell'estrema concretezza del suo processo di adattamento alla realtà. [...] In questa prospettiva cambia radicalmente anche il modo di porsi nei confronti delle immagini, le quali non vengono più semplicemente ridotte ad una presunta logica nascosta, celata sotto le mentite spoglie, ad esempio, dell'immagine onirica. Per Jung le immagini sono un punto di partenza e non di arrivo. (Cicali \& Squilloni, 1994)

Nella sua psicologia archetipica, Hillman (1984) riprende questa visione e la amplifica: per lui le immagini sono la psiche stessa. In tal senso, egli definisce la "poetica della mente», "il fare dell'anima tramite l'immaginazione delle parole» (ivi, p. 63): «[...] questo 'fare' è poiesis. Conoscere la profondità della mente significa conoscere le sue immagini» (ivi, p. III). E dunque: «[...] il fondamento poetico della mente ci dice che la logica selettiva che opera nelle trame delle nostre vite è la logica del mythos, è mitologia» (ivi, p. 13).

Così anche Sacks (2001) che, da neurologo, da tempo ha descritto l'organizzazione iconica dell'esperienza e di ogni cognizione della stessa: «[...] l'esperienza non è possibile finché non è organizzata iconicamente [...]. La 'registrazione cerebrale' di ogni cosa - di ogni cosa viva - deve essere iconica. è questa la forma finale della registrazione cerebrale, anche se la forma preliminare può essere computazionale o programmatica [...]» (ivi, p. 199).

Nella medesima direzione erano già le ricerche di Arnheim (1974) relativamente al pensiero visivo: "I concetti sono immagini percettive e [...] le operazioni del pensiero sono il trattamento di tali immagini» (ivi, p. 267), dunque concetto e percetto sono "due aspetti di un'unica e medesima esperienza» (ivi, p. 321).

Il linguaggio iconico, dunque, è via di accesso alla realtà, non sua preclusione (Lumer \& Zeki, 2011). Gregory Bateson, già molto prima delle validazioni giunte dall'utilizzo della risonanza magnetica funzionale (Lucignani \& Pinotti, 2007; Cappelletto, 2009), scriveva che la metafora è la forma stessa della realtà. Ed è forma della realtà in quanto è forma della stessa conoscenza umana:

Se vogliamo parlare di esseri viventi, non solo in veste di biologi accademici, ma di esseri viventi a nostra volta, in mezzo a esseri viventi, sarebbe opportuno adottare un linguaggio che fosse in qualche modo isomorfo, che fosse coerente con il linguaggio in base al quale gli esseri viventi stessi sono organizzati. (Bateson, 1997, p. 458) 


\section{E dunque:}

[...] la metafora non è solo una belluria poetica, non è logica buona o logica cattiva, ma è di fatto la logica con cui è stato costruito il mondo biologico, è la principale caratteristica e la colla organizzativa di questo mondo del processo mentale. (Bateson \& Bateson, 1989, p. 53)

Nella visione batesoniana, la metafora comprende tutti i processi di conoscenza e comunicazione che ineriscono asserzioni di somiglianza, analogia, combinazione, ovvero: al cospetto di qualsiasi fenomeno ignoto - sia esso un oggetto, una circostanza, una relazione - la conoscenza di esso e la comunicazione con esso muove attraverso un confronto per sovrapposizione (Bateson, 1984 , p. 112) e, dunque, per combinazione/analogia/somiglianza con gli aspetti noti presenti nella interna ecologia delle idee, per cui il conoscere è un cogliere/stabilire nessi.

Conoscere è, allora, sempre un'operazione metaforica ed, ergo, simbolica: conoscere come tessere, legare, connettere. Ove il cogitare si rivela, appunto, come co-gitare: agitare insieme (Faenza, 2005, p. 380).

[...] nel mondo vi sono state, e ancora vi sono, molte epistemologie, diverse e addirittura contrastanti, che hanno però sostenuto tutte l'idea di un'unità di fondo e, benché ciò sia meno certo, hanno anche sostenuto l'idea che questa unità di fondo è estetica. L'uniformità di questi pareri fa sperare che forse la grande autorità della scienza quantitativa non basti per negare l'idea di una bellezza unificatrice fondamentale. Io mi attengo al presupposto che l'aver noi perduto il senso dell'unità estetica sia stato, semplicemente, un errore epistemologico. (Bateson, 1984, p. 34)

Unità estetica. Visione sistemica. E che è, appunto, il proprium di ogni metafora, ove sfondo e figura, forma e sostanza, si saldano, e ove, pertanto, il mythos non dice di meno o male della realtà ma, anzi, ne dice la verità più profonda, potendo accedere, nel suo regime notturno, a ciò che è precluso al regime diurno proprio del logos (Durand, 1972). E se ciò avviene, chiarisce Morin, è per via del particolare isomorfismo tra mito e vita, in ragione di ciò che entrambe le contraddistingue e, persino, le fonda e ne costituisce l'identità: la comune natura metamorfica.

Il mito è metamorfico come l'evoluzione biologica, il che significa che quest'ultima somiglia terribilmente al mito. (Morin, 1993a, p. 182)

È questa modalità metaforica e metamorfica di conoscenza che Bateson configura precipuamente come sensibilità estetica (Bateson, 1997, p. 389 ss.): coscienza ecologica delle connessioni (Manghi, 2004), delle interdipendenze, dei legami, anche tra ciò che apparentemente sembra appartenere a mondi diversi. 
E per questo egli investe la metafora di un ruolo cruciale a livello epistemico e ontologico, considerandola la struttura che tutto connette - mente e natura, microcosmo e macrocosmo, parti e configurazioni, classi e predicati -, fondamento di quella "grammatica creaturale» che consente «nuovi modi di guardare e di pensare agli organismi e ai prati, e anche agli esseri umani» (Bateson $\&$ Bateson, 1989, p. 289). Nuovi modi: estetici, sintetici, binoculari ${ }^{1}$.

\section{RAZIONALITÀ STEREOSCOPICA}

Metaforizzare è dunque la coscienza di quell'ecologia che svela che nulla è slegato, come nelle parole del poeta: «[...] nulla è, tutto coesiste» (Pessoa, 1986, p. 259).

La metafora, ponte biologico tra i linguaggi dei nostri due emisferi cerebrali (Lakoff \& Johnson, 1998), è dunque non solo modalità di conoscenza (Munari, 1993, pp. 23-39) ma anche, e soprattutto, in quanto esercizio/addestramento alla complessità, scuola di flessibilità e palestra cerebrale. Essa si configura, così, come via epistemologica ed, insieme, didattica: la via che sceglie i linguaggi della metafora, dell'analogia e del simbolo è, difatti, via che riconosce le potenzialità metabletiche del linguaggio artistico, il suo potere di "ridescrivere l'esperienza, di ristrutturare la realtà» (Ricoeur, 2001, p. XXIII).

Metaforizzare, dunque, operazione mentale e finanche "fisica»: prodigio di sottrazione dalla propria epifenomenicità, in ragione della umana capacità di elaborare non solo fatti e informazioni presenti davanti a noi qui ed ora - $\mathrm{i}$ percetti -, ma anche, e soprattutto, dati assenti dal nostro campo percettivo eppur creati/prodotti/realizzati da noi stessi - le immagini mentali -. Sicché, quando il bambino non ha un cavallo vero e neppure uno a dondolo, quella assenza/mancanza/limitazione genera una simbolizzazione: cavalcare una scopa immaginandola un destriero. Percezione e immagini mentali, pur funzionando in maniera simile, non sono identiche: giacché mentre «i percetti visivi sono stabili» - «in quanto riflettono la realtà circostante» - «le visualizzazioni interne sono mutevoli» (Kosslyn, 1999, p. 121) - perché noi ne siamo i creatori -.

1 «L'immagine binoculare, che appare indivisa, è in realtà una complessa sintesi, compiuta dall'emisfero destro, di informazioni provenienti dall'emisfero sinistro e una corrispondente sintesi, compiuta dall'emisfero sinistro, di materiale proveniente dall'emisfero destro. Successivamente questi due aggregati di informazioni sintetizzate vengono a loro volta sintetizzati in una singola immagine soggettiva dalla quale è scomparsa ogni traccia di demarcazione verticale. [...] Con questo nuovo genere di informazione l'osservatore aggiunge alla visione un'ulteriore dimensione» (Bateson, 1984, p. 98). 
Sono, allora, nelle nostre "visualizzazioni interne» - nelle nostre metafore, nei simboli che ci appartengono, nei miti che ci descrivono, nella rappresentazione che scegliamo per rispecchiarci e vederci da fuori (Zeki, 2011) - le nostre possibilità di trasformazione (Watzlawick, 1986). Ovvero: di transito $\rightarrow$ passaggio $\rightarrow$ evoluzione da una «vecchia forma» a una nuova, che è lo specifico della formazione. Esse sono, allora, «conoscenza metamorfica»: poiché in esse "il conoscere è un pathos che modifica il soggetto conoscente ...» (Calasso, 1991, p. 491).

Nei contesti della formazione scientifica, la metaforizzazione è dunque non solo artificio letterario ma anche e soprattutto sfondo epistemologico e esercizio didattico che consente la promozione della competenza del saper uscire dalle proprie cornici (Sclavi, 2002) - routines consolidate di pensiero e azione (Mortari, 1998) - giacché:

Con le immagini mentali possiamo pensare ciò che abbiamo visto e trasformarlo a nostro piacimento. A volte queste trasformazioni sono deliberate, come quando cerchiamo di visualizzare il modo migliore di sistemare le valigie nel bagagliaio dell'auto, oppure ci mettiamo a fantasticare su eventi assolutamente improbabili [...]. E questo uno dei caratteri distintivi della vita mentale: la facilità con cui si possono creare scene mai esistite o trasformare il banale quotidiano in un evento straordinario. Se vogliamo sperare di rispondere agli interrogativi circa i modi in cui la mente registra e utilizza l'informazione, dobbiamo capire la nostra capacità di considerare l'ipotetico - di costruirci un'immagine del mondo non semplicemente com'è, ma come potrebbe essere. (Kosslyn, 1999, pp. 121-122)

È questa capacità di trasformazione il tratto distintivo della capacità rappresentativa (Piaget, 1967) che quando, verso il diciottesimo mese di vita, comincia a consentirci di trasformare l'immagine materna in una immagine interiore da richiamare in sua assenza, ci introduce nel mondo del possibile, che non è il mondo dell'artificio ma, anzi, è il medesimo mondo donde nasce la scienza: l'immaginazione è reale in quanto ricade nella realtà, agisce nella datità, feconda il tangibile, non solo da esso si astrae. Poiché è un movimento di andata-e-ritorno dal così-come-è verso il come-può-essere. Ed è un movimento che è insieme cognitivo e operativo, meta-fisico e fisico, giacché genera comportamenti, non solo meditazioni (Zeki, 2000 e 2008).

L'arte non è dunque soltanto contenuto, oggetto, disciplina ma metafor$m a$, epistemologia e insieme procedura che genera conoscenza: e ciò perché origina situazioni in grado di provocare «uno scompenso, una rottura del flusso ordinario di percezione» (Dallari, 2001, p. 32) giacché, nei setting di formazione, proprio come nella ricerca scientifica, è lo «scompenso» che genera apprendimento (Bateson, 2000). 
Dalla psicologia archetipica, dunque, attraversando la storia della scienza (Hanson, 1978; Hofstadter, 1996) e la storia dell'estetica (Perniola, 1997) fino alle indagini di neurobiologia, neuroestetica e psicologia costruttivista ${ }^{2}$, discende una visione dell'immaginario che, diversamente dalla sua interpretazione razioide ${ }^{3}$ (Musil, 1979) non lo considera pericoloso e illusorio, «illegittimo e destabilizzante poiché impedisce l'accesso alla verità» in quanto privo di "alcun fondamento razionale» (Certini, 2004, p. 38) ma riconosce la nostra duplice identità di Savant e Rêveur: soggetto-che-conosce e soggettoche-immagina (Bachelard, 1972), ove l'immaginazione non è né subalterna né alternativa alla conoscenza: poiché l'immaginare non spodesta il ragionare ma, anzi, lo corrobora.

E l'immaginazione, allora, non è il contrario dell'azione, giacché, anzi, muove-spinge-crea, non soltanto contempla.

L'immaginazione è, difatti, "messa in scena» di quel "teatro interno», tanto psichico quanto somatico, che Jung aveva identificato come motore del cambiamento ereditando le intuizioni di Ignazio di Loyola:

Si attribuisce ai Gesuiti il merito di aver introdotto il teatro nella normale prassi educativa. Ma bisogna anche riconoscere al Loyola, con i suoi Exercitia, la geniale proposta di un uso del «teatro interno» [...].

Per la salvezza dell'anima datevi all'arte - sembra essere il monito di Loyola datevi al teatro! Anzi, datevi al cinema, perché negli Exercitia la visione ha il posto d'onore: essa è l'indispensabile riscontro della parola. Ma, a scanso di equivoci, sarebbe più esatto dire "fate del cinema!», e fatelo da protagonisti, perché Loyola non vuole un popolo di spettatori.

Non si tratta infatti di ricevere passivamente, perché è nel fare, è nel responsabilizzarsi che l'anima ritrova la salute. Con gli Exercitia il sogno viene sottratto al monopolio del regime notturno che lo vuole passivo e insensato.

È il caso di dire che alle fasi REM notturne, cioè alle allucinate visioni, alle possessioni oniriche della notte, Loyola oppone le fasi REM diurne, ispirate costruzioni che animano i sensi e l'intelletto: «sogni» che devono scandire il ritmo della giornata quasi con la stessa cadenza con cui i sogni colorano la notte.

2 Cfr. Bruner, 1976 e 1994; Kosslyn, 1980 e 1994; Kanizsa, 1985 e 1991; Ugazio, 1988; Watzlawick, 1988; Massironi, 1988; Hubel, 1989; Gregory, 1991; Ceruti \& Preta, 1998; Robertson, 2003; Zeki, 2003, 2008 e 2011; Solms \& Turnbull, 2004; Varisco, 2004; Cappelletto, 2009; Lumer \& Zeki, 2011.

3 «L'uomo razioide è l'uomo che 'persegue' gli schemi rigidi e monotoni della razionalità univoca, che disarticola il mondo in cose separate, in frammenti, le cui operazioni cognitive e pratiche non consistono in altro se non nella incessante e monotona ripetizione di un meccanismo concettuale che da sempre è predisposto, come un antefatto che anticipa ogni nostra possibile mossa o impresa intellettuale, che ricorre costantemente per bisogno di sicurezza a uno stile univoco di pensiero, che cerca di esorcizzare il nuovo e l'originale» (Musil, 1979, p. 6). 
Il sogno va dunque sottratto all'incoscienza del sonno, al suo torpore che vorrebbe requisirlo: esso va restituito alla piena lucidità del giorno.

Certo, si sa che il giorno è sempre percorso da un lungo serpeggiante sogno clandestino. Ma il daydreaming è per i più un sognare disimpegnato che si sfrangia e si disperde.

Loyola esige che questa eccezionale risorsa sia disciplinata e potenziata. Da occasionale, deve farsi sistematica, deve tematizzarsi e approfondirsi. E deve prendere consistenza ed evidenza tali da gareggiare con le immagini del mondo esterno.

Dunque, come il sogno è attraversato da temi ricorrenti, così anche la lucidità della veglia deve avere i suoi «sogni», le sue ritmiche accensioni oniriche. Non le fragili, scolorite, vaganti emergenze,gli abbozzi per lo più informi del daydreaming, ma una presenza salda, un tessuto coerente.

Una realtà tanto vivida nel suo apparire quanto profonda nel suo significato.

Realtà sensibile e intellegibile: sogno al lume della ragione. (Faenza, 2005, pp. 362-363)

L'epistemologia che sottende questa consapevolezza riprende, pertanto, quella visione di «arte come esperienza» che Dewey (1951) indicava per sottolineare la valenza formativa della creazione e della fruizione artistica.

Non già forme della leggerezza, dunque, le espressioni simboliche (Zeki, 2000, 2008 e 2011). Bensì forme della conoscenza. E dunque della formazione.

\section{INTERSTIZI DELLA CONOSCENZA (E DELLA FORMAZIONE)}

«L'arte e la scienza sono parimenti mezzi per comprendere» (Goodman, 1984, p. 108):

[...] supporre che la scienza sia piattamente e pedestramente linguistica, letterale e denotazionale vorrebbe dire non tenere conto, ad esempio, di aspetti quali l'uso frequente di strumenti analogici, della metafora implicita nella misurazione quando uno schema numerico viene applicato a un nuovo campo, o del vocabolario corrente della fisica e dell'astronomia, in cui si parla di attrazione, di singolarità e di buchi neri. Anche se il prodotto ultimo della scienza è, a differenza di quello dell'arte, una teoria letterale, verbale, matematica, denotazionale, la scienza e l'arte procedono di pari passo nella loro ricerca e nella loro costruzione. (Goodman, 1978, pp. 125-126)

"Contrapporle le fraintende e le danneggia entrambe» (Goodman, 1968, p. 5) - perché l'una e l'altra custodiscono «un carattere fondamentalmente cognitivo» (ivi, p. 211) e, pertanto, esse costituiscono modi, sebbene dif- 
ferenti, per il perseguimento del medesimo obiettivo: «[...] far avanzare la conoscenza» (Goodman, 1984, p. 4).

Così Goodman celebra l'unità degli opposti, intuendo, ben prima delle validazioni empiriche giunte dalla neurobiologia, come e quanto la competenza scientifica non sia antitetica a quella estetica.

E tale percezione e celebrazione della complessità ${ }^{4}$ - per cui complexus è ciò che, con Morin, riconosciamo come "tessuto insieme» (Morin, 1988, p. 56) - a suggerire una epistemologia della formazione scientifica che nei linguaggi simbolici trova razionalità, non evasione.

Razionalità il cui sguardo si fa stereoscopico, razionalità che sistemicamente tesse, di contro ad una che disgiunge, razionalità «interpretabile nei termini di un inter-legere piuttosto che di un intus-legere» (Carmagnola, 2000, p. 140).

Alla luce di tali riflessioni, è in corso una microsperimentazione che prevede la formazione "estetica» dei dottorandi in Pedagogia e Scienze Pedagogiche presso l'Università di Foggia ${ }^{5}$, promuovendo seminari di crossing disciplinare (insieme alle discipline pedagogiche, i dottorandi si formano agli statuti e alle metodologie di un pattern composito di ambiti di ricerca accademica: antropologia, sociologia, filosofia, storia della scienza, estetica) e atelier tra forme, metaforme e contenuti dei singoli saperi, per promuoverne abduzioni, intrecci, ibridazioni (Scardicchio, 2012a e 2012b).

Promuovere la competenza estetica nella formazione dottorale implica l'esercizio dello sguardo morfologico: giacché il morfologo è, appunto, colui che indaga le connessioni, le somiglianze, i nessi, anche tra ciò che, all'apparenza, e alla logica formale, sembrerebbe dissimile, sconnesso, lontano ${ }^{6}$.

In tal modo, nella ricerca e nella formazione si recupera il valore di quei "contenuti laterali» che la scienza laplaciana aveva scartato, considerandolo inutili e, finanche, antitetici alla conoscenza. E li si riscopre non in quanto sussidiari ma, persino, preziosi elementi indiziari (Ginzburg, 1979) della verità.

Ed è proprio con questa attenzione agli indizi che provengono anche da altre logiche, che si sviluppa la competenza dell'abdurre (Eco \& Sebeok, 1983; Bateson, 1984 e 1989; Peirce, 1984; Bonfantini, 1986) di quella «ab-

4 Cfr. von Bertalanffy, 1971; Hofstadter, 1984; Prigogine, 1986; Bocchi \& Ceruti, 1988; Maturana \& Varela, 1992; Cerrato et al., 1996; Benkirane, 2007; De Toni \& Comello, 2007.

5 Il corso di dottorato, di cui è coordinatore Isabella Loiodice, ordinario di Pedagogia Generale presso l'Università di Foggia, afferisce a: École Doctorale Internationale. Culture, Éducation, Communication - Universités Lisbonne - Paris X - Potsdam - Roma Tre - Avignon et Pays Vaucluse - Foggia.

6 Cfr. Morin, 1983, p. 117; Black, 1983; Thom, 1980; Hofstadter, 1984. 
duzione creativa» (Eco, 1990, p. 238) che si caratterizza, come nella originaria indicazione di Pearce, come la capacità di spiegare un oggetto sconosciuto muovendo da qualcosa di conosciuto, anche se da esso distante, nell'intreccio tra noto e ignoto, familiare ed estraneo.

Bateson descrive l'abduzione come «il procedimento con cui da fenomeni appartenenti a campi diversi si estrae ciò che hanno in comune» (Bateson \& Bateson, 1989, p. 65), capacità di cogliere i nessi, correlare, pensare esteticamente concetti, oggetti, proposizioni:

Possiamo esaminare l'anatomia di una rana e poi guardarci intorno per scoprire altri esempi delle stesse relazioni astratte ricorrenti in altre creature, compresi, in questo caso, noi stessi. Questa estensione laterale delle componenti astratte della descrizione è chiamata abduzione ... (Bateson, 1984, p. 192)

Ogni abduzione può essere vista come una descrizione doppia o multipla di qualche oggetto o evento o sequenza. Se esamino l'organizzazione sociale di una tribù australiana e lo schema delle relazioni naturali su cui è basato il totemismo, vedo che questi due blocchi di conoscenza stanno tra loro in relazione abduttiva, cioè obbediscono entrambi alle stesse regole. In ciascun caso si suppone che certe caratteristiche formali di una componente siano riflesse nell'altra. (ivi, pp. 192-193)

Una delle possibili ricadute di tale visione «doppia o multipla» - che è estetica proprio perché coglie abduttivamente le correlazioni, le analogie, i nessi tra mondi distinti - è il crossing disciplinare (Licata, 2010): quella preparazione trans-disciplinare che consente al ricercatore di trovare una via in pedagogia studiando chimica, trovare una soluzione in medicina attraverso la storia dell'arte. Molteplici sono, nella storia e nella filosofia della scienza, le scoperte riconducibili a questa capacità di traslazione, a questa competenza nella trasduzione di mondi eppure, ancora, le si ritiene il frutto di una disposizione individuale, della esperienza singola di uno scienziato "più creativo" di un altro, e non già la metaforma precipua della logica e della scoperta scientifica, così come dell'apprendimento e, dunque, della didattica.

In tale direzione binoculare, potrebbero altresì essere progettati percorsi di formazione per i giovani ricercatori, non soltanto centrati sui subjects disciplinari ma fondati su una visione metaforica $\rightarrow$ sistemica $\rightarrow$ estetica. Giacché estetica non è la forma "romantica» o "suggestiva» della scienza e della formazione ma è la forma stessa della realtà (e, dunque, della conoscenza): irriducibilmente doppia.

E la «descrizione doppia» è il processo epistemologico che consente di superare l'autoreferenzialità della visione monoculare, propria di ogni singolo osservatore/singola osservazione disciplinare, e di accedere a una visione che è binoculare poiché interroga la realtà dalle posizioni/osservazioni di più 
epistemologie. Ovvero: nel processo di conoscenza/interpretazione, l'epistemologia «a zig zag» di Bateson com-prende, non già separa - come invece da procedura sperimentale precipua della scienza moderna - analitico e sintetico, concetto e immagine, realtà e sua metaforizzazione, considerate nel loro indissolubile intreccio poiché il loro esistere è un esistere costituzionalmente intrecciato: tessuto insieme $\rightarrow$ ecologico $\rightarrow$ simbolico.

Cogliere i nessi, inter-legere, simbolizzare si rivela dunque la competenza estetica di ogni scienza e di ogni ricercatore. Ed è così - ovvero: muovendo abduttivamente e non già per fortuità o caso - che il ricercatore può sperare nella serendipità (Merton \& Baber, 2002); Colazzo la identifica come la capacità di profezia retrospettiva, indicando la competenza basilare di una mente che flessibilmente si muove tra possibilità, plurilogicità, complessità, scevra dal bisogno di matematizzare il reale:

Serendipità ed abduzione giocano un ruolo di fondamentale importanza nell'ambito della scienza, che non può essere né il risultato di un processo puramente induttivo (come erroneamente riteneva Bacone), né appagarsi delle tautologie (come ben aveva intuito già Kant) delle procedure deduttive. Necessita dell'intervento della sagacia del ricercatore, cioè della sua inventiva e della sua creatività. Solo così è possibile che egli trovi ciò che non cerca ed individui ingegnose soluzioni a problemi impossibili. La serendipità ha evidentemente a che fare col principio, enunciato da Feyerabend, della «sovrabbondanza del reale», che è il rovesciamento dell'enunciazione hegeliana di coincidenza di razionale e reale. (Colazzo, 2005, pp. 5-6)

Ed è in questo rovesciamento che, schiudendo «le porte dell'interpretazione meta-logica del reale», si dischiudono le porte dell'interpretazione meta-logica della ricerca e della formazione. Ove, l'utilizzo dei linguaggi «altri» e delle metafore ed analogie nella procedura di analisi, così come di comprensione dei fenomeni, giunge ad essere considerato «non più radice inconfessabile ma naturale veicolo per la costruzione del sapere scientifico» (Carmagnola, 1989, p. 236).

Abduzione, serendipità, analisi morfologica: competenze storicamente dentro l'intuizione scientifica, eppure storicamente fuori dai suoi percorsi di apprendistato. La sperimentazione in corso si prefigge, altresì, di offrire ai dottorandi un percorso transisciplinare che consenta loro di acquisire non solo subjects e metodologie disciplinari, non solo contenuti né soltanto forme, ma un approccio estetico alla conoscenza e, dunque, alla ricerca scientifica, che si connoti, proprio come nella indicazione batesoniana, verso la costruzione di una «scienza delle relazioni»:

Coloro che danno più importanza alle «cose» che stanno in relazione ( $\mathrm{i}$ «termini della relazione») respingeranno ogni analogia tra grammatica e anatomia botanica, considerandola troppo arzigogolata; dopo tutto una foglia e un so- 
stantivo non si rassomigliano affatto nell'apparenza esteriore. Ma se poniamo le relazioni in primo piano e ne consideriamo i termini come definiti unicamente da quelle, allora cominciamo ad avere qualche dubbio. Esiste un'analogia profonda tra grammatica e anatomia? Esiste una scienza interdisciplinare che dovrebbe occuparsi di tali analogie? (Bateson, 2000, pp. 193-194)

Una scienza interdisciplinare che si occupa di analogie - tra mondi, linguaggi, discipline - non implica la rinuncia alla conoscenza oggettiva ma l'approdo a una oggettività, intrisa di simbolico tanto quanto di analitico, che non oppone scientifico e poetico giacché considera il secondo, tanto quanto il primo, una interpretazione/modellizzazione del reale, "una visione della realtà dal punto di vista della 'interfaccia linguaggio/vivente'” (Carmagnola, 1989 , p. 239) e, dunque, riconosce e utilizza entrambe come forme e metaforme della conoscenza ed, ergo, della ricerca scientifica.

E così il Franzini può affermare che «l'estetico è dunque l'orizzonte precategoriale di ogni possibile e reale indagine scientifica [...]. E l'estetica è la scienza di questo precategoriale, cioè il territorio fondazionale di ogni formazione epistemologica» (Franzini, 2007, p. 163).

Ed ecco perché al ricercatore sono richieste le medesime posture dell'esteta (Scardicchio, 2012a): di colui che conosce nella implicazione e nella ibridazione, non già nell'astrazione e nella assolutezza. Ed ecco perché la sua formazione evoca pratiche complesse e composite in cui il crossing disciplinare consenta il superamento delle logiche di categorizzazione - e scissione - dei saperi e, indipendentemente dalla specializzazione disciplinare di ogni dottorato, che sia "esatta» o "umana», la scienza a cui abilitarsi coniughi esteticamente province confinanti tra arte e scienza (Koestler, 1975), scorgendo e legittimando parentele insospettate (Damasio, 1987, p. 265) tra saperi provenienti da domini diversi (Prigogine \& Stengers, 1981).

È questa la specificità dello sguardo "estetico» alla costruzione del percorso formativo (Gallerani, 2012) evocato da ogni disciplina accademica ove, con Calvino, si possa riconoscere che poiché «la scienza diffida dalle spiegazioni generali e dalle soluzioni che non siano settoriali e specialistiche», allora "la grande sfida» - della scienza, non solo della letteratura - è il comprendersi e descriversi come «sistema di sistemi» e, allora, imparare a "tessere insieme i diversi saperi e i diversi codici in una visione plurima, sfaccettata del mondo» (Calvino, 1988, p. 110) e della conoscenza. E dunque della formazione.

Sicché i piani di formazione dottorale possano attingere dalla chimica come dalla poesia, dall'epistemologia come dalla letteratura, dalla ingegneria come dall'arte figurativa, dalla medicina come dal teatro, costruendo un percorso di educazione scientifica che non sia antitetica a quella artistica (Dewey, 1951; Perniola, 2007; Russo, 2007) e nulla dei modi «umani» del conoscere risulti loro estraneo. 
Sarà così possibile per l'Accademia progettare percorsi di formazione estetica - nel suo senso batesoniano - per i propri ricercatori: percorsi a «doppio pensiero» (Minichiello, 1994) nei quali tessere le singole aree disciplinari nel tessuto più ampio che connette Bohr a Calvino, Feynman a Magritte, Einstein a Fellini, accettando lo scompenso che deriva da ogni meticciamento, considerato breccia e non spaccatura.

Modi di pensare postdarwiniani (Bocchi \& Ceruti, 1984) che si sostanziano, con le parole di Morin: «[...] nell'arte [...] di concepire le interazioni, le interferenze, e gli intrecci polisistemici» (Morin, 1983, p. 181).

Come da indicazione moriniana dunque, questa estetica progettazione della formazione scientifica «richiede una ristrutturazione della configurazione complessiva del sapere» e dunque il fondamento di una «Scienza Nuova» (Morin, 1993b, p. 48):

Non si tratta solo di stabilire relazioni diplomatiche e commerciali tra le discipline, dove ognuna si confermi nella sua sovranità. Si tratta di mettere in causa il principio di discipline che mutilano con l'accetta l'oggetto complesso, il quale è costituito essenzialmente dalle interrelazioni, le interazioni, le interferenze, le complementarità, le opposizioni tra elementi costitutivi ciascuno dei quali è prigioniero di una disciplina particolare. (Morin, 1994, p. 111)

Da Husserl alla fisica dei quanti, da Gödel a Bach, da Heisenberg ad Escher, da Weil a Mozart, da Merleau Ponty a Picasso, l'umana avventura della conoscenza passa attraverso la coscienza della irriducibilità della coesistenza del simbolico e dell'analitico - universale e particolare, eternità e tempo, indicibile e dicibile - sebbene ciò risulti illogico per quella vecchia scienza la cui antica etimologia la vuole - dalla radice skei - signora della divisione (Bröcker, 2004).

Sicché alla Scienza Nuova, al crocevia per statuto (Morelli \& Weber, 2010) è chiesto d'esser "non soltanto critica, ma autocritica»: "[...] si riconosce la vera razionalità dalla sua capacità di riconoscere le proprie insufficienze» (Morin \& Kern, 1994, p. 166).

\section{RIFERIMENTI BIBLIOGRAFICI}

Arnheim, R. (1974). Il pensiero visivo. La percezione visiva come attività conoscitiva. Torino: Einaudi.

Bachelard, G. (1972). La poetica della rêverie. Bari: Dedalo.

Bateson, G. (1984). Mente e natura. Un’unità necessaria. Milano: Adelphi.

Bateson, G. (1997). Una sacra unità. Milano: Adelphi. 
Bateson, G. (2000). Verso un'ecologia della mente. Milano: Adelphi.

Bateson, M. C., \& Bateson, G. (1989). Dove gli angeli esitano. Verso una epistemologia del sacro. Milano: Adelphi.

Benkirane, R. (2007). La teoria della complessità. Torino: Bollati Boringhieri.

Bertalanffy, L. von (1971). Teoria generale dei sistemi. Fondamenti, sviluppo, applicazioni. Milano: Isedi.

Black, M. (1983). Modelli archetipi metafore. Parma: Pratiche.

Bocchi, G., \& Ceruti, M. (1984). Modi di pensare postdarwiniani. Saggio sul pluralismo evolutivo. Bari: Dedalo.

Bocchi, G., \& Ceruti, M. (a cura di). (1988). La sfida della complessità. Milano: Feltrinelli.

Bröcker, M. (2004). Parte-del-mondo: la posizione di Heinz von Foerster. Rivista Europea di Terapia Breve Strategica e Sistemica, 1.

Bruner, J. (1976). Psicologia della conoscenza. Roma: Armando.

Bruner, J. (1994). Il conoscere. Saggi per la mano sinistra. Roma: Armando.

Calasso, R. (1991). I quarantanove gradini. Milano: Adelphi.

Calvino, I. (1988). Lezioni americane. Milano: Garzanti.

Cappelletto, C. (2009). Neuroestetica. L'arte del cervello. Roma - Bari: Laterza.

Carmagnola, F. (1989). La visibilità. Per un'estetica dei fenomeni complessi. Milano: Guerini.

Carmagnola, F. (2000). La specie poietica. Teorie della mente e intelligenza sociale. Milano: Mimesis eterotopia.

Cerrato, S., Zanarini, G., Virasoro, M., Codenotti, B., Zellini, P., \& De Luca, A. (1996). Caos e complessità. Napoli: Cuen.

Certini, R. (a cura di). (2004). L'immaginario: una "frontiera avanzata" della formazione e della scuola. Roma: Carocci.

Ceruti, M., \& Preta, E. (a cura di). (1998). Che cosè la conoscenza. Roma - Bari: Laterza.

Cicali, C. A., \& Squilloni, D. (1994). L'immagine e l'icona. http://www.centroicone.it/ bollettino\% 204. htm.

Colazzo, S. (2005). Formare al pensiero abduttivo con Webquest. In V Congresso SIRD La ricerca didattica per la formazione degli insegnanti. http://sdefad. uniroma3.it/sird/vari/I/Colazzo.pdf.

Dallari, M. (2001). Arte e stupore infantile. In M. A. Donna (a cura di), Tra scuola e museo: lo spazio dell'arte. Torino: Città di Torino - Divisione Servizi Culturali.

Damasio, A. (1987). L'errore di Cartesio. Emozione, ragione e cervello umano. Milano: Adelphi.

Dewey, J. (1951). Arte come esperienza. Firenze: La Nuova Italia. 
Durand, G. (1972). Le strutture antropologiche del'immaginario. Introduzione all'archetipologia generale. Bari: Dedalo.

Faenza, V. (2005). L'arte di curare con l'arte. Discorsi di psicoterapia. Milano: Guaraldi. Feyerabend, P. K. (1984). Scienza come arte. Roma - Bari: Laterza.

Franzini, E. (2007). Immagine e pensiero. In G. Lucignani \& A. Pinotti (a cura di), Immagini della mente. Neuroscienze, arte e filosofia. Milano: Raffaello Cortina.

Gadamer, H. G. (1983). Verità e metodo. Lineamenti di un'ermeneutica filosofica. Milano: Bompiani.

Gallerani, M. (2012). L'educazione permanente: un'ipotesi/proposta per lo sviluppo dell'empowerment individuale e sociale. Pedagogia più Didattica, 3, 11-20.

Ginzburg, C. (1979). Spie. Radici di un paradigma indiziario. In A. Gargani (a cura di), Crisi della ragione. Torino: Einaudi.

Goodman, N. (1968). The languages of art. An approach to a Theory of Symbols. Indianapolis: Bobbs-Merrill.

Goodman, N. (1978). Ways of worldmaking. Indianapolis: Hackett Publishing.

Goodman, N. (1984). Of mind and other matters. Cambridge, MA: Harvard University Press.

Gregory, R. L. (1991). Occhio e cervello. La psicologia del vedere. Milano: Raffaello Cortina.

Hillman, J. (1984). Le storie che curano. Milano: Raffaello Cortina.

Hofstadter, D. R. (1984). Gödel, Escher, Bach: un'eterna ghirlanda brillante. Milano: Adelphi.

Hofstadter, D. R. (1996). Concetti fluidi e analogie creative. Modelli per calcolatore dei meccanismi fondamentali del pensiero. Milano: Adelphi.

Hubel, D. H. (1989). Occhio, cervello e visione. Bologna: Zanichelli.

Jung, C. G. (1965). Opere, Vol. 5: Simboli della trasformazione. Torino: Bollati Boringhieri.

Kanizsa, G. (1985). Grammatica del vedere. Saggi su percezione e Gestalt. Bologna: Il Mulino.

Kanizsa, G. (1991). Vedere e pensare. Bologna: Il Mulino.

Koestler, A. (1975). L'atto della creazione. Roma: Ubaldini.

Kosslyn, S. M. (1994). Le immagini nella mente. Firenze: Giunti.

Lakoff, G., \& Johnson, M. (1998). Metafora e vita quotidiana. Milano: Bompiani.

Licata, I. (2010). Leggi di protezione, analogie e metafore interattive. La ragionevole. efficacia del crossing disciplinare. Riflessioni Sistemiche, 3, 16-24.

Lucignani, G., \& Pinotti, A. (a cura di). (2007). Immagini della mente. Neuroscienze, arte e filosofia. Milano: Raffaello Cortina.

Lumer, L., \& Zeki, S. (2011). La bella e la bestia: arte e neuroscienze. Roma - Bari: Laterza. 
Manghi, S. (2004). La conoscenza ecologica. Milano: Raffaello Cortina.

Marcuse, H. (1968). Eros e civiltà. Torino: Einaudi.

Massironi, M. (1988). Fenomenologia della percezione visiva. Bologna: Il Mulino.

Minichiello, G. (1994). Il doppio pensiero. Razionalità e analogia nel discorso pedagogico. Napoli: Morano.

Morelli, U. (2010). Mente e bellezza. Arte, creatività e innovazione. Torino: Alemmandi.

Morelli, U., \& Weber, C. (2010). Conflitto della conoscenza. Vincoli e possibilità dell'interdisciplinarità. Riflessioni Sistemiche, 3, 35-47.

Morin, E. (1983). Il metodo. Ordine, disordine, organizzazione. Milano: Feltrinelli.

Morin, E. (1988). Le vie della complessità. In G. Bocchi \& M. Ceruti (a cura di), La sfida della complessità. Milano: Feltrinelli.

Morin, E. (1993a). La conoscenza della conoscenza. Milano: Feltrinelli.

Morin, E. (1993b). Introduzione al pensiero complesso. Milano: Sperling e Kupfer.

Morin, E. (1994). Il paradigma perduto. Che cos'è la natura umana? Milano: Feltrinelli.

Morin, E., \& Kern, A. B. (1994). Terra-Patria. Milano: Raffaello Cortina.

Mortari, L. (1998). Ecologicamente pensando. Milano: Unicopli.

Perniola, M. (2007). Come leggere "Art as experience» nel quadro dell'orizzonte estetico attuale? In L. Russo (a cura di), Esperienza estetica a partire da John Dewey. Palermo: Aesthetica.

Piaget, J. (1967). La formazione del simbolo nel bambino. Imitazione, gioco e sogno. Immagine e rappresentazione. Firenze: La Nuova Italia.

Prigogine, I. (1986). Dall'essere al divenire. Tempo e complessità nelle scienze fisiche. Torino: Einaudi.

Prigogine, I., \& Stengers, I. (1981). La nuova alleanza. Metamorfosi della scienza. Torino: Einaudi.

Ricoeur, P. (2001). La metafora viva. Dalla retorica alla poetica: per un linguaggio di rivelazione. Milano: Jaka Book.

Robertson, I. H. (2000). L'occhio della mente. Milano: Rizzoli.

Sacks, O. (2001). L'uomo che scambiò sua moglie per un cappello. Milano: Adelphi.

Scardicchio, A. C. (2011). Didactiques entre légèreté et profondeur: les ateliers d'artjeu-autobiographie. In I. Loiodice, P. Plas, \& N. Rajadell, Université et formation tout au long de la vie. Un partenariat européen de mobilité sur les thèmes de l'éducation des adultes. Paris: L'Harmattan.

Scardicchio, A. C. (2012a). Il sapere claudicante. Per una estetica della ricerca e della formazione. Milano: Bruno Mondadori.

Scardicchio, A. C. (2012b). Logica \& Fantastica. "Altre» parole nella formazione. Pisa: ETS. 
Scholem, G. (1986). Le grandi correnti della mistica ebraica. Genova: Il Melangolo.

Sclavi, M. (2002). Arte di ascoltare e mondi possibili. Milano: Le Vespe.

Solms, O., \& Turnbull, M. (2004). Il cervello e il mondo interno. Milano: Raffaello Cortina.

Ugazio, V. (a cura di). (1988). La costruzione della conoscenza. Milano: Franco Angeli.

Varisco, B. M. (2004). Costruttivismo socio-culturale. Genesi filosofiche, sviluppi psicopedagogici, applicazioni didattiche. Roma: Carocci.

Watzlawick, P. (1986). Il linguaggio del cambiamento. Milano: Feltrinelli.

Watzlawick, P. (a cura di). (1988). La realtà inventata. Milano: Feltrinelli.

Zeki, S. (2000). La visione dall'interno. Arte e cervello. Torino: Bollati Boringhieri.

Zeki, S. (2008). Con gli occhi del cervello. Roma: Di Renzo.

Zeki, S. (2011). Splendori e miserie del cervello. Milano: Codice.

\section{Riassunto}

Lo scritto muove da una riflessione intorno alle due forme della conoscenza umana - analitica e simbolica - e da li si interroga a proposito del loro utilizzo congiunto nella scienza e nella formazione scientifica dei ricercatori, di qualsivoglia disciplina o ambito accademico. La conoscenza scientifica viene dunque considerata come irriducibilmente fondata sul "crossing disciplinare», verso la proposta di una "estetica della formazione del ricercatore»: ove l'«estetica» corrisponde alla "sensibilità sistemica", indicata da Bateson come quella conoscenza "ecologica" in grado di cogliere nessi-congiunzioni-incroci tra saperi, linguaggi e metodi provenienti da domini diversi, verso la costruzione di una "scienza interdisciplinare che si occupa di analogie». L'approdo è verso quella "Scienza Nuova» - che Morin riprende da Vico - caratterizzata da abduzione, serendipità, valorizzazione degli elementi storicamente considerati «laterali» nella ricerca, laddove " $i$ modi di pensare postdarwiniani» si traducono in progettazioni formative estetiche: binoculari, complesse, sistemiche. In tal senso, la capacità di «descrizione doppia» - l'epistemologia a zig zag indicata da Bateson - delinea l'isomorfismo tra competenze del ricercatore - di qualsivoglia disciplina - $e$ postura estetica.

Parole chiave: Conoscenza scientifica, Crossing disciplinare, Estetica, Formazione, Visione sistemica. 